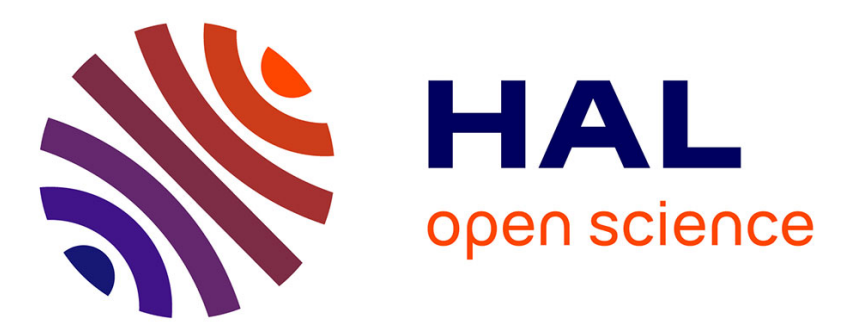

\title{
Intention de fidélité à la marque des participants à une communauté virtuelle de marque: le rôle dual de l'engagement
}

Marie-Laure Gavard-Perret, K. Raies

\section{To cite this version:}

Marie-Laure Gavard-Perret, K. Raies. Intention de fidélité à la marque des participants à une communauté virtuelle de marque: le rôle dual de l'engagement. 2011, 32 p. halshs-00640092

\section{HAL Id: halshs-00640092 \\ https://shs.hal.science/halshs-00640092}

Submitted on 10 Nov 2011

HAL is a multi-disciplinary open access archive for the deposit and dissemination of scientific research documents, whether they are published or not. The documents may come from teaching and research institutions in France or abroad, or from public or private research centers.
L'archive ouverte pluridisciplinaire HAL, est destinée au dépôt et à la diffusion de documents scientifiques de niveau recherche, publiés ou non, émanant des établissements d'enseignement et de recherche français ou étrangers, des laboratoires publics ou privés. 


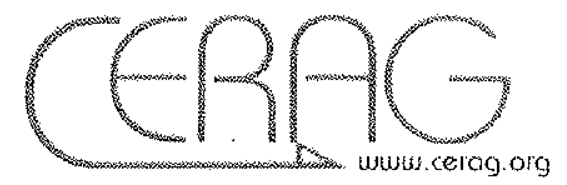

Centre d'Eudes et de Recherches Rpptiquées ó lo Gestion UMA CNA.5. 5890

\section{CAHIER DE RECHERCHE $n^{\circ}$ 2011-01 E3}

Intention de fidélité à la marque des participants à une communauté virtuelle de marque : le rôle dual de l'engagement

\section{Karine RAIES}

Docteur en Marketing, Enseignant Chercheur ESC Saint Etienne

Laboratoire CERAG UMR CNRS 5820

51,53 cours Fauriel, 4200 Saint Etienne

\section{Marie-Laure GAVARD-PERRET \\ Professeur des Universités \\ IAE de Grenoble - Université de Grenoble \\ BP 47, 38040 Grenoble \\ Laboratoire CERAG UMR CNRS 5820}


Intention de fidélité à la marque des participants à une communauté virtuelle de marque : le rôle dual de l'engagement

\author{
Karine Raïes \\ Docteur en Marketing, Enseignant Chercheur ESC Saint Etienne \\ 51,53 cours Fauriel, 42000 Saint Etienne \\ karine_raies@esc-saint-etienne.fr
}

\begin{abstract}
Marie-Laure Gavard-Perret
Professeur des Universités

IAE de Grenoble - Université de Grenoble

BP 47, 38040 Grenoble Cedex 9

Laboratoire CERAG - UMR CNRS 5820
\end{abstract}

marie-laure.gavard-perret@upmf-grenoble.fr 
Intention de fidélité à la marque des participants à une communauté virtuelle de marque : le rôle dual de l'engagement

RESUME

Dans un contexte de développement de l'utilisation des médias sociaux, il convient de mieux comprendre l'effet de la participation des consommateurs aux communautés organisées autour de marques sur la relation de ces derniers avec les marques concernées. Ainsi, cette recherche examine, à travers une étude quantitative auprès de 1065 membres d'une communauté virtuelle autour de la marque Nikon, l'effet de l'intensité de leur participation à la communauté sur leurs intentions de fidélité envers la marque Nikon ainsi que l'effet médiateur de leur engagement envers la communauté dans cette relation. De plus, la relation de type bidirectionnel qu'entretiennent l'engagement envers la communauté et l'engagement envers la marque est examinée et confirmée. Enfin, le rôle modérateur de l'ancienneté est souligné.

Mots Clés : Médias sociaux, forums de discussion, communauté virtuelle de marque, intention de fidélité à la marque, engagement envers la communauté, engagement envers la marque, modèle non récursif bi-directionnel.

Brand Loyalty Intention of virtual brand community members : the dual role of commitment

ABSTRACT

In a context of growing use of social media, it is useful to better understand the effect of participation of consumers to communities structured around brands on the relation between the consumers and the concerned brands. This research examines, through a quantitative study among 1065 members of a Nikon's community, the effect of intensity of consumer participation in a virtual brand community on brand loyalty intentions firstly, and the mediator role of community commitment in this relationship, secondly. The bidirectional relationship between community commitment and brand commitment is examined and confirmed. A positive moderator role of membership duration is underlined.

Keys words: social media, discussion forum, virtual brand community, brand loyalty intention, community commitment, brand commitment, non-recursive bidirectional model. 


\section{INTRODUCTION}

En 1999 déjà, Levine et alii avançaient, de manière assez avant-gardiste, que «les entreprises qui ne comprennent pas que leurs marchés sont désormais un réseau d'individus à individus, très impliqués dans un dialogue et par conséquent plus intelligents, passent à côté de leur meilleure chance ». Depuis, ces réseaux de consommateurs partageant des informations sur les produits et services se sont développés de manière exponentielle avec la démocratisation des médias sociaux. Ainsi, une étude menée par le CREDOC en $2009^{1}$ indique que $57 \%$ des internautes français consultent l'avis d'autres internautes sur les produits et services qu'ils souhaitent acheter. De même, un sondage d'Harris Interactive ${ }^{2}$ montre que $64 \%$ des internautes américains ayant acheté un produit sur Internet déposent un avis sur l'utilisation de ce produit.

Ces constats ont retenu l'attention des entreprises, nombreuses à avoir compris le pouvoir que représentent les médias sociaux dans la diffusion de leur image. Le succès de certains blogs de marques et pages de passionnés de marques (fans pages) sur Facebook (par exemple, la marque Ferrero classée première avec 4,147 millions d'inscrits ${ }^{3}$ ) en est la preuve. L'intégration des médias sociaux dans les considérations et stratégies marketing est ainsi devenue une question majeure pour les entreprises. Dans cette perspective, il importe de mieux comprendre l'impact que la participation à ces relations et discussions en ligne entre consommateurs peut avoir sur leurs intentions envers les marques concernées. Cet objectif général a guidé la présente recherche.

Dans la mesure où, au cour du développement des médias sociaux se trouve le phénomène communautaire, il est judicieux de porter plus particulièrement l'attention sur le cas de la communauté virtuelle autour d'une marque donnée, communauté qui peut être initiée soit par des consommateurs intéressés par la marque, soit par la marque elle-même. En l'occurrence, la présente recherche se référera au premier type de communauté, créée à l'initiative de consommateurs qui se retrouvent autour de leur intérêt commun pour une marque (ici la marque Nikon) à travers des discussions en ligne (forums de discussion). Ce type de rassemblement pourrait être identifié, sur la base de la classification des regroupements via les médias sociaux proposée par Kaplan et Haenlein (2010), comme un projet collaboratif ${ }^{4}$.

Or, si les études pionnières qui se sont intéressées à ces consommateurs membres de communautés ont clairement soutenu l'idée que ces regroupements méritent l'attention des chercheurs aussi bien que des managers (Amine et Sitz, 2004; De Valck, 2004 ; Bagozzi et Dholakia, 2006b; Cova, 2006; etc.), peu d'études en revanche ont démontré empiriquement un effet de la participation du consommateur à des communautés virtuelles autour d'une marque (CVM) sur son attitude et/ou son comportement envers la marque concernée. Seules quelques

\footnotetext{
${ }^{1}$ http:/www.journaldunet.com/cc/01 internautes/inter usage fr.shtml

${ }^{2} \mathrm{http}: /$ www.deiworldwide.com/files/DEIStudy-Engaging\%20ConsumersOnline-Summary.pdf

${ }^{3} \mathrm{http}: / / \mathrm{fr}$-fr.facebook.com/Nutella?ref =ts en septembre 2010.

${ }^{4}$ Les projets collaboratifs sont définis par Kaplan et Haenlein (2010) comme « des projets permettant la mise en commun et la création simultanée de contenus par les utilisateurs $»$.
} 
rares études ont examiné le rôle de cette participation du consommateur sur la fidélité, à travers un effet direct (Shang et alii, 2006; Li et Hung, 2006) ou à travers le rôle médiateur de la confiance (Casalo et alii, 2007; Ellonen et alii, 2010).

De plus, alors même que les investissements conduits et/ou les partenariats instaurés par certaines grandes marques dans le cadre de ces outils communautaires sont explicitement ou implicitement guidés par l'espoir de se rapprocher de leurs consommateurs, de gagner leur confiance et d'augmenter ainsi leur engagement et leur fidélité envers la marque (Armstrong et Hagel, 1996), la variable " engagement »-pourtant reconnue dans la littérature pour ses liens forts avec la fidélité (Dholakia, 1997; Amine, 1998; Pritchard et alii, 1999; Fullerton, 2005)n'a, quant à elle, pas encore trouvé sa place dans les recherches relatives aux communautés virtuelles de marque. Il est donc particulièrement pertinent de mesurer et de comprendre l'effet de la participation du consommateur à une communauté virtuelle autour d'une marque sur sa fidélité envers la marque, sous l'angle de la double forme d'engagement impliquée dans ce cas : l'engagement envers la communauté, d'une part et l'engagement envers la marque, d'autre part. Dans cette perspective, plutôt que de comparer les effets de la participation à une CVM à ceux d'une non-participation ou encore à ceux de différents types de participation, problématiques déjà explorées par d'autres auteurs (par exemple, Butler et alii, 2002; McAlexander et alii, 2003 ; Shang et alii, 2006; Casalo et alii, 2007), il apparaît plus intéressant de vérifier l'influence de l'intensité de la participation des membres de la communauté sur la relation qu'ils entretiennent avec la marque. Une participation soutenue et intense d'un membre à une communauté est en effet de nature à renforcer son engagement envers cette dernière, et ce pour de multiples raisons : au travers des liens affectifs et/ou sociaux qu'il est susceptible de développer via ses échanges avec les autres membres de la communauté; au travers des considérations utilitaires qu'il peut avoir à l'égard de la communauté (répondre par exemple à ses besoins informationnels); en raison de son sentiment d'utilité (par ses propres apports aux autres membres) ; etc. Par ailleurs, les auteurs qui défendent l'intérêt des communautés virtuelles de marque et leur impact sur le renforcement de la relation avec la marque soutiennent l'idée de l'effet positif de cet engagement envers la communauté sur l'intention de fidélité à la marque (Algesheimer et alii, 2005; Heeyoung et alii, 2008). La présente recherche aura donc pour premier objectif de vérifier l'incidence de l'intensité de participation à une CVM, -variable sur laquelle la marque peut essayer d'agir directement par des apports de contenu, l'animation de concours, etc.--, sur l'engagement envers la communauté d'une part, et l'effet de cet engagement sur l'intention de fidélité à la marque, d'autre part. L'engagement, considéré ici comme la volonté du participant à continuer sa relation avec le partenaire selon la définition de Moorman et alii (1993), renvoie, d'après Allen et Meyer (1990), à la nature même du lien qui unit le participant à l'organisation concernée, en l'occurrence la CVM.

De plus, ainsi que précisé précédemment, les entreprises qui implantent ou sont partenaires de communautés autour de leur marque sont le plus souvent guidées par leur volonté d'accroître la fỉdélité des consommateurs envers leur marque au travers des satisfactions et bénéfices procurés par la communauté à ses membres. En augmentant, par ces actions, l'engagement envers la CVM, elles espèrent influer favorablement sur l'engagement envers la marque. Or, si l'intensité de participation des membres à la communauté semble influencer positivement leur engagement envers cette communauté (comme évoqué plus tôt), et s'il n'est plus à démontrer 
que l'engagement envers la marque peut être considéré comme un antécédent de l'intention de fidélité envers cette dernière, la relation qu'entretient l'engagement envers la communauté avec l'engagement envers la marque dans ce contexte communautaire mérite d'être plus précisément examinée. Ce sera le second objectif de cette recherche.

Pour répondre à ces questions, une étude a été menée auprès de 1065 membres d'une communauté virtuelle autour de la marque Nikon initiée par un consommateur : "Les Pixélistes ». Les membres échangent grâce à un forum de discussion très actif et des activités diversifiées (poser des questions, apporter de l'aide aux débutants, poster des photos, voter pour des photos, échanger autour de la marque et de ses produits, organiser des sorties, etc.). Les résultats de cette étude seront présentés après avoir, dans la première partie, posé le cadre conceptuel de ce travail et en avoir dégagé un corps d'hypothèses, et dans une deuxième partie, exposé la méthodologie retenue pour tester le modèle conceptuel proposé. A la suite de l'analyse des résultats seront soulignées les contributions du présent travail, puis certaines de ses limites seront mises en avant et enfin, des voies de recherche proposées.

\section{CADRE CONCEPTUEL ET HYPOTHESES}

De la participation à une communauté virtuelle de marque (CVM) à la fidélité envers la marque: rôle de l'engagement envers la communauté

Les auteurs ayant défendu l'intérêt, pour l'entreprise, des communautés virtuelles autour d'une marque sont nombreux (Aubert-Gamet et Cova, 1999; Arnould et Price, 2000; Muniz et O'Guinn, 2001; Gupta et Kim, 2004); leurs recherches restent cependant, le plus souvent, théoriques. Le besoin, compréhensible, de mieux cerner et maîtriser ce sujet encore nouveau a, jusqu'à présent, plutôt conduit à des approches exploratoires. Les études qualitatives, notamment ethnographiques, ont ainsi été très sollicitées dans ce domaine dès les années 1990 (Schouten et McAlexander, 1995; Cova, 1997; Schau et Muniz, 2002; Amine et Sitz, 2004). Pourtant, quelques recherches empiriques portant sur des CVM sont apparues avec les travaux précurseurs de McAlexander et alii (2003). Ces auteurs démontrent une relation positive entre l'intégration du consommateur dans une communauté et sa satisfaction ainsi que sa fidélité vis-à-vis du service offert par l'entreprise. De même, Shang et alii (2006) ainsi que Li et Hung (2006) ont testé l'effet de la participation des consommateurs à une CVM sur la fidélité à la marque et confirment les résultats des travaux précédents. Enfin, une étude menée par Casalo et alii (2007) démontre l'effet positif de la participation à des CVM relatives à des logiciels informatiques sur la confiance et la fidélité envers la marque.

Cependant, ces premiers résultats soutenant l'existence d'un effet positif de la participation du consommateur à une CVM sur sa relation avec la marque ne se situent jamais dans un contexte français et très rarement dans un contexte européen. De plus, la majorité de ces recherches se sont limitées soit à la participation versus non-participation du membre (DuttaBergman, 2005, Algesheimer et Dholokia, 2006), soit au niveau de participation en termes de fréquence et/ou durée de participation (Algesheimer et alii, 2005 ; Langerak et alii, 2003). La 
présente recherche se donne pour objectif d'aller au delà en considérant, en plus de la simple participation, l'effet de l'intensité de contribution à la CVM. En effet, il est plausible de penser que plus le consommateur contribue aux échanges, discussions et activités qui ont cours au sein d'une CVM construite autour d'une marque, plus son intention de fidélité envers cette marque est susceptible d'en être positivement affectée. Il est alors plus judicieux d'aborder, et c'est le choix fait dans cette recherche, l'intensité de participation au travers de deux facettes différentes, comme le font Wang et Fesenmaier (2004) : 1- le niveau de participation en termes de fréquence et de durée moyenne, qui ne reflète que le fait d'être plus ou moins présent ; 2- le niveau de contribution, fonction du nombre d'activités auxquelles le membre prend part au sein de la CVM.

D'autre part, au delà de l'étude de l'impact de l'intensité de la participation du membre à une communauté virtuelle de marque sur son intention de fidélité envers la marque, il est essentiel de comprendre le mécanisme d'influence de cette participation sur la fidélité à la marque en mettant en évidence le rôle médiateur joué dans cette relation par l'engagement envers la communauté. L'engagement envers la communauté, identifié comme la volonté de maintenir sa relation avec la communauté, a été introduit pour la première fois dans le domaine des communautés virtuelles de marque par Gupta et Kim (2007). Ces auteurs considèrent alors que l'engagement envers une communauté a comme antécédent une participation intense du membre à la communauté. De leur côté, Algesheimer et alii (2005) défendent l'idée que l'intention de continuer à être membre de la communauté, autrement dit l'engagement envers la communauté, a pour conséquence des intentions de comportement envers la marque. Ces résultats sont par ailleurs confirmés par les travaux de Heehyoung et alii (2008) qui démontrent une relation positive entre l'engagement envers la communauté et la fidélité à la marque, cet engagement étant lui-même influencé par la participation à la communauté.

Il est ainsi possible de poser la première hypothèse stipulant que plus l'intensité de la participation du membre à la CVM est élevée, plus son lien avec cette communauté et sa volonté de continuer sa relation avec cette dernière (autrement dit son engagement envers la communauté) seront forts et plus son intention de fidélité avec la marque, objet du rassemblement de la CVM, sera fort, d'où le postulat du rôle médiateur de l'engagement envers la communauté :

H1 : L'engagement envers la communauté est une variable médiatrice de la relation entre l'intensité de participation à la CVM et l'intention de fidélité à la marque.

Afin de préciser cette relation de médiation, et conformément aux recommandations de Baron et Kenny (1986), l'hypothèse H1 sera donc décomposée comme suit :

- H1.a : L'intensité de participation à la CVM a un impact positif sur l'intention de fídélité à la marque 
- H1.b : L'intensité de participation à la CVM a un impact positif sur l'engagement envers la communauté

- H1.c: L'engagement envers la communauté a un impact positif sur l'intention de fidélité à la marque.

Un engagement double, à l'égard de la communauté et à l'égard de la marque

L'engagement relationnel a été identifié par Moorman et alii (1993) comme étant "le désir de continuer la relation avec le partenaire ». En ce sens, dans le cadre d'une communauté, l'engagement renvoie à une facette psychologique et affective de la relation que construit le consommateur avec la communauté. Or, si l'on se réfère aux travaux de Kozinets (1999), la relation d'un individu avec une communauté se fonde sur deux dimensions : sa relation avec la communauté et sa relation avec l'objet d'intérêt de la communauté (ici la marque). L'objectif de ce travail de recherche étant de mieux comprendre la construction de la fidélité du consommateur envers la marque dans le contexte particulier des communautés virtuelles autour de marques, il semble tout à fait pertinent de ne pas se limiter à une seule forme d'engagement mais de prendre en compte les liens qu'il peut y avoir entre, d'une part l'engagement envers la CVM et, d'autre part l'engagement envers la marque, marque qui est l'objet de rassemblement de la CVM.

Une relation bidirectionnelle entre l'engagement envers la communauté et l'engagement envers la marque

Dans le contexte spécifique qui est au centre de ce travail, la relation que va construire le consommateur avec les membres de la CVM est susceptible d'avoir un impact sur sa relation avec l'objet du rassemblement. Cette relation a d'ailleurs été mise en exergue par Bagozzi et alii (2006b) qui montrent que la relation du consommateur avec la communauté de marque a un impact positif sur sa relation avec la marque. D'autre part, il semble également évident qu'un engagement envers la marque plus fort va nourrir l'engagement du membre envers la communauté construite autour de cette marque. Allant dans ce sens, De Almeida et alii (2008) concluent que l'attachement à la marque a un effet positif sur la relation avec la communauté.

La littérature ne statue donc pas précisément sur le sens de la relation entre le lien construit par le membre avec la communauté et celui qu'il construit avec la marque. Ce constat est appuyé par les propos de Kozinets (1999) qui parle de deux variables «non indépendantes » ef "interreliées", que sont la relation du membre avec la communauté et sa relation avec la marque. Il semblerait par conséquent que cette relation soit à double sens. Ainsi, un engagement envers la marque plus fort nourrirait l'engagement envers la communauté, de même qu'un engagement plus fort envers la communauté influencerait à son tour de manière positive 
l'engagement envers la marque. Ce constat permet d'avancer la deuxième hypothèse :

H2: L'engagement envers la communauté et l'engagement envers la marque s'influencent réciproquement.

Ce type de relation, identifiée comme une relation en boucle, peut être modélisé grâce à des modèles non récursifs bidirectionnels, introduits par Duncan et alii (1968) en sciences sociales et par Bagozzi (1980) en marketing. Sur cette base, l'hypothèse H2 se décompose comme suit :

- H2a : L'engagement envers la marque influence positivement l'engagement envers la communauté.

- H2b : L'engagement envers la communauté influence positivement l'engagement envers la marque.

La relation engagement envers la marque et intention de fidélité

Deux approches de la fídélité peuvent être identifiées dans la littérature. La première approche, purement comportementale, définit et mesure la fidélité par le comportement de rachat de la marque (Cunnigham, 1961; Odin, 1998; Johnson et alii, 2006); la seconde, plus relationnelle, s'intéresse à la relation qui se construit entre le consommateur et la marque et introduit l'idée que la fidélité ne peut exister sans engagement (Morgan et Hunt, 1994 ; Oliver, 1999 ; Pritchard et alii, 1999 ; Garbarino et Johnson, 1999). Divers travaux faisant référence à la fidélité attitudinale identifient l'engagement comme moyen de différencier vraie et fausse fidélité (pour une revue, voir Garnier, 2006). L'engagement envers une marque représente alors la volonté du sujet de rester client de la marque sur la base d'un attachement et d'une identification envers celle-ci (Moorman et alii, 1993) et est, à ce titre, souvent vu comme une variable médiatrice clé dans la formation de la fidélité (Morgan et Hunt, 1994; Garbarino et Johnson, 1999; Pritchard et alii, 1999). Sur la base de ces travaux, il est donc légitime de considérer l'engagement envers la marque comme un antécédent de l'intention de fidélité.

Cela amène à poser la troisième hypothèse :

H3 : L'engagement envers la marque a un impact positif sur l'intention de fidélité à la marque. 
Effet modérateur de l'ancienneté d'appartenance à la communauté :

Le rôle de la durée d'appartenance à la communauté a été reconnu par différents auteurs comme étant important dans la prédiction des attitudes et comportements de ses membres (De Valck, 2004; Langerak et alii, 2003; Rothaermel et Sugiyama, 2001). Par ailleurs, Alon et Brunel (2007) exposent les différentes phases par lesquelles peut passer le membre d'une communauté virtuelle de marque depuis son inscription. Selon eux, le type de relation que développe le membre passe d'une relation rationnelle basée sur l'échange d'information (task oriented) à une relation socio-émotionnelle basée sur des conversations intimes, le développement d'affinités et le partage d'expériences communes, influençant ainsi le développement d'un engagement affectif envers la communauté. De même, ce rôle d' "ancien » au sein de la communauté, rôle reconnu et respecté par les autres membres, peut amener le participant à se sentir plus "obligé » vis-à-vis des autres membres et donc jouer sur son engagement normatif envers la communauté. Enfin, l'ancienneté de participation est susceptible de modérer positivement le lien entre l'engagement du membre envers la communauté et sa relation avec la marque. Ainsi, plus un membre est ancien, plus son engagement envers la communauté aura un impact positif sur son engagement envers la marque et son intention de fidélité envers celle-ci. Il est donc raisonnable de supposer que l'ancienneté d'appartenance jouera un rôle favorable, conformément à l'hypothèse suivante.

H4: L'ancienneté de l'appartenance à la communauté a un effet modérateur positif sur l'ensemble des relations qui existent entre le niveau de participation à la CVM et l'intention de fidélité à la marque.

La figure 1 reprend ces hypothèses et formalise le modèle de recherche qui sera testé.

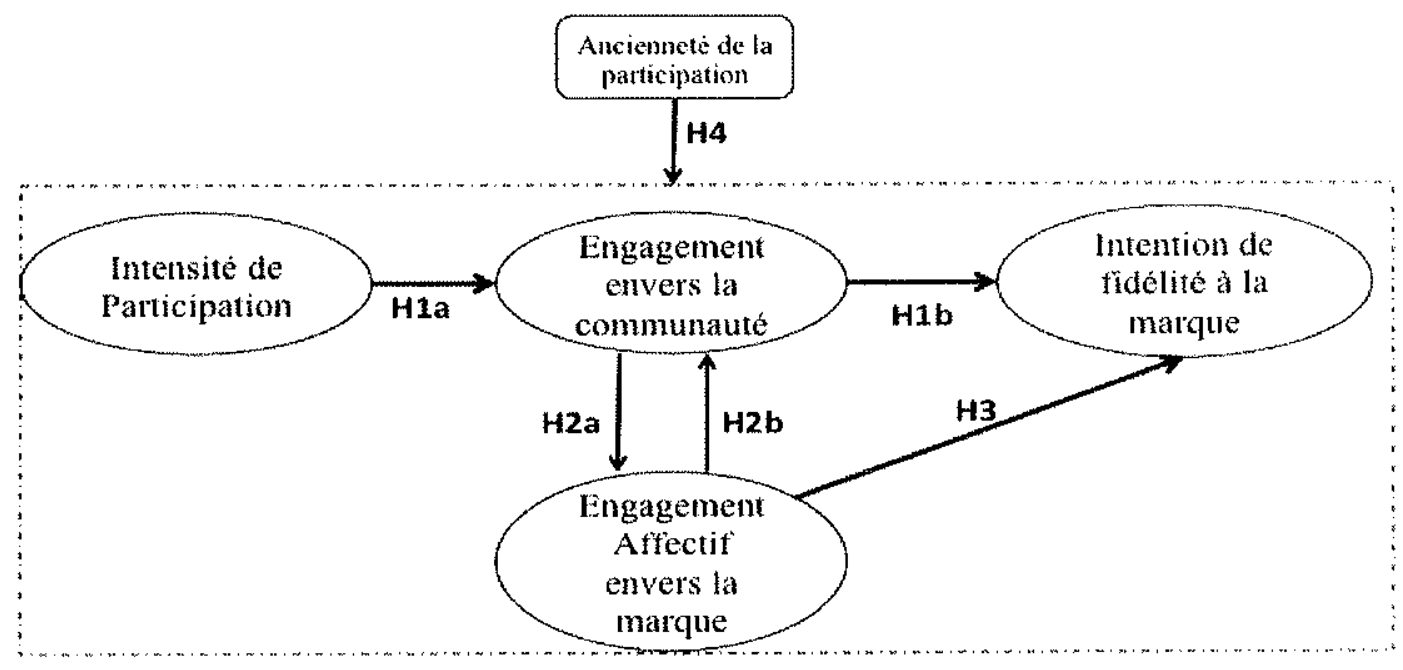

Figure 1 - Modèle de Recherche 


\section{METHODOLOGIE DE LA RECHERCHE}

\section{Le terrain d'étude : un forum de discussion autour de la marque Nikon}

Afin de tester le modèle de recherche qui s'est dégagé du cadre conceptuel présenté (figure 1), un terrain a été mis en place en Janvier 2008 en partenariat avec une communauté virtuelle existante «Les pixélistes » initiée par un consommateur suite à l'achat d'un appareil photo Nikon et organisée autour de cette marque célèbre. Présentée alors sur Internet comme «la première communauté de Nikonistes Francophone $"$, elle comptait près de 30000 membres enregistrés et Nikon comme partenaire principal. La communauté «Les Pixélistes » permet à des consommateurs d'interagir grâce à Internet (et plus particulièrement à travers un forum de discussion) et de partager leur intérêt pour la marque Nikon en échangeant connaissances, expériences, avis, sentiments à propos de cette marque et plus largement leur passion pour la photographie. Cette communauté, dont la modération se fait de manière indépendante de la marque, est néanmoins en contact avec le service marketing de cette dernière qui l'informe des nouveautés ou des offres du moment, voire organise des concours de photographie. Un logo et un lien vers le site commercial sont d'ailleurs présents sur le site de la communauté.

Une étude quantitative auprès des membres du forum de discussion de cette CVM a ainsi été réalisée, avec l'appui de Nikon France qui, à cette époque, ne possédait pas encore de forum de discussion sur son propre site Internet et était curieuse de savoir si cela pourrait être intéressant de mettre ce type d'outil à la disposition des consommateurs. Depuis l'étude, un espace communautaire virtuel autour de la marque $\left(M y N^{\prime} c l u b\right)$ est venu compléter les dispositifs de la Nikon School (dispositif de formation des Nikonistes à travers conseils et stages) et du Nikon club (communauté réelle permettant l'organisation de sorties entre passionnés de la photo).

Un questionnaire a ainsi été mis en ligne sur le site du forum avec une brève présentation de l'objectif de l'étude par le modérateur du forum. Ont été obtenues par cette voie 1065 réponses utilisables (après élimination des réponses aberrantes et des questionnaires incomplets). Le choix des échelles de mesures utilisées dans le questionnaire est exposé dans la section suivante.

\section{Choix des mesures}

Afin de mesurer l'engagement envers la communauté, la mesure qui a semblé être la plus adéquate pour rendre compte de la volonté de maintenir sa relation avec une communauté mais aussi de toutes les composantes de ce lien est la mesure multidimensionnelle du concept développée dans un contexte organisationnel par Allen et Meyer (1990). Il est en effet possible 
de retrouver dans cette mesure les dimensions généralement évoquées par la littérature ayant traité des communautés de marque mais également issues de nos premières investigations qualitatives, à savoir : (a) une dimension affective de la relation (présente notamment dans les travaux de Kang et alii en 2007 qui mettent en avant l'importance du sentiment d'appartenance et de l'attachement psychologique), l'intérêt de rester membre de la communauté reconnu comme un engagement calculé (qui est évoqué dans le contexte des CVM par Casalo et alii (2008) et qui a été révélé dans la phase d'observation de discours de la communauté préalable à l'enquête au travers de verbatim tels que : "heureusement que vous êtes là, je ne peux plus me passer de vos conseils!") et (b) un sentiment d'obligation envers les autres membres de la communauté (qui renvoie au « sens de la responsabilité morale » identifié par Muniz et O'Guinn (2001)). Afïn de valider l'adaptabilité à la mesure de l'engagement envers une communauté virtuelle de marque de cette échelle de mesure créée dans un environnement organisationnel, un prétest a été effectué auprès de 150 participants à une communauté virtuelle d'intérêt ( « chatmania »), pré-test qui a permis d'épurer la mesure et d'adapter certains items au contexte communautaire.

La mesure de l'intensité de la participation à la communauté s'inspire des travaux de Langerack et alii (2004) qui mesurent le niveau de participation par la durée (Combien de temps en moyenne dure chaque visite de la $C V M$ ) et par la fréquence (combien de fois en moyenne visitez-vous la CVM) et de Shang et alii (2006) et Wang et alii (2004) qui ajoutent aux mesures précédemment évoquées celle du niveau de contribution du membre aux activités de la communauté co $^{5}$ Sur la base des travaux de Jarvis et alii (2003) qui mettent en garde face à la mauvaise spécification des modèles qui ne distinguent pas les variables latentes de type formatif de celles de type réflectif ${ }^{6}$, il est important de préciser que la variable "intensité de participation $»$ telle que mesurée dans cette recherche, et composée de trois items : la fréquence de participation, la durée de participation et le niveau de contribution, est une variable formative.

Ensuite, pour mesurer l'intention de fidélité envers la marque Nikon, le choix a été fait d'une mesure centrée sur la dimension comportementale de la fidélité : l'intention de racheter et de recommander la marque, dans la mesure où a déjà été intégrée dans le modèle la variable " engagement envers la marque », considérée sous son angle relationnel et souvent vue comme la facette affective de la fidélité. En conséquence, sont empruntés, pour la mesure de l'intention de fidélité comportementale, les items proposés par Johnson et alii (2006) prenant en compte l'intention de rachat d'une marque ( 3 items) et le bouche-à-oreille positif ( 2 items).

\footnotetext{
${ }^{5}$ La variable «niveau de contribution » a été mesurée à travers une échelle à 4 modalités : 1 - pour les personnes ayant coché uniquement «lire les messages et s'informer», 2- pour les participants ayant coché une ou deux activités, 3-pour les participants ayant coché trois ou quatre activités, 4- pour ceux ayant coché plus de quatre activités différentes. Les différentes activités recensées auprès des participants sont : Poser des questions à d'autres membres sur le forum, répondre aux questions des autres membres, participer aux concours des Pixelistes, passer/répondre à une annonce vente, participer à des sorties avec d'autres membres Pixelistes, commenter les photos des autres membres Pixelistes, poster des messages à propos de la marque Nikon.
}

${ }^{6}$ Pour plus de détails sur les règles permettant de distinguer les variables de type formatif des variables de type réflectifí, voir l'article de Jarvis et alii (2003). 
Enfin, pour l'engagement affectif envers la marque, susceptible d'exprimer l'aspect relationnel de la fidélité envers la marque et reconnu comme étant la volonté d'un individu de rester client de la marque sur la base d'un attachement et d'une identification envers celle-ci, c'est la mesure de l'engagement affectif proposé par Fullerton (2005) qui sera reprise. Tous les instruments de mesure utilisés dans cette recherche ont fait l'objet de pré-tests et d'analyses factorielles (en composantes principales) pour vérifier leurs qualités psychométriques.

\section{Validation des échelles de mesure}

L'analyse en composante principale a permis d'épurer les échelles de mesure utilisées. Est ainsi obtenue, pour l'engagement envers la communauté, une mesure à trois dimensions qui permet de restituer plus de $75 \%$ de la variance et dont le coefficient alpha de Cronbach de chaque dimension est supérieur à 0,8 (voir Annexe Al). Par la méthode des équations structurelles (grâce au logiciel AMOS), il est possible de confirmer la bonne qualité psychométrique de cette échelle, avec un RMSA de 0,059, un AGFI de 0,958 et un CFI de 0,983. La fiabilité des dimensions de l'échelle est établie avec un Rhô de Joreskog supérieur à 0,6 (sauf pour la dimension engagement d'obligation où il est égal à 0,598 ). Enfin, la validité convergente est vérifiée avec un indice supérieur à 0,5 , ce qui signifie que la variance de chaque construit est davantage expliquée par ses mesures que par l'erreur (Fornell et Larcker, 1981). Les résultats de l'AFC de l'échelle de mesure de l'engagement envers la communauté sont résumés dans le tableau 1. 


\begin{tabular}{|c|c|c|c|c|}
\hline Item & $\lambda$ & $\begin{array}{c}\lambda \\
\text { bootstrap }\end{array}$ & $\begin{array}{c}\text { Ecart } \\
\text { type }\end{array}$ & Valeur \\
\hline $\begin{array}{l}\text { Engagement communautaire affectif } \\
\text { COMAFF1 } \\
\text { COM_AFF2 } \\
\text { COM_AFF3 } \\
\text { COM AFF4 }\end{array}$ & $\begin{array}{l}0,845 \\
0,825 \\
0,775 \\
0,765\end{array}$ & $\begin{array}{l}0,846 \\
0,824 \\
0,777 \\
0,767\end{array}$ & $\begin{array}{l}0,013 \\
0,015 \\
0,017 \\
0,021\end{array}$ & $\begin{array}{l}65,075 \\
54,933 \\
45,705 \\
36,523\end{array}$ \\
\hline Rhô de Jöreskog & \multicolumn{4}{|c|}{0,878} \\
\hline Validité convergente & \multicolumn{4}{|c|}{0,860} \\
\hline $\begin{array}{l}\text { Engagement communautaire calculé } \\
\text { COM_CAL1 } \\
\text { COM_CAL2 }\end{array}$ & $\begin{array}{l}0,596 \\
0,963\end{array}$ & $\begin{array}{l}0,595 \\
0,962 \\
\end{array}$ & $\begin{array}{l}0,028 \\
0,024 \\
\end{array}$ & $\begin{array}{c}21,25 \\
40,083\end{array}$ \\
\hline Rhô de Jöreskog & \multicolumn{4}{|c|}{0,772} \\
\hline Validité convergente & \multicolumn{4}{|c|}{0,641} \\
\hline $\begin{array}{l}\text { Engagement communautaire obligation } \\
\text { COM_OBL1 } \\
\text { COM_OBL2 }\end{array}$ & $\begin{array}{l}0,579 \\
0,725\end{array}$ & $\begin{array}{l}0,577 \\
0,725\end{array}$ & $\begin{array}{l}0,030 \\
0,035\end{array}$ & $\begin{array}{l}12.233 \\
21.428\end{array}$ \\
\hline Rhô de Jöreskog & \multicolumn{4}{|c|}{0,598} \\
\hline Validité convergente & \multicolumn{4}{|c|}{0,530} \\
\hline $\begin{array}{l}\text { RMSEA } \\
\text { AGFI } \\
\text { GFI } \\
\text { CFI } \\
\text { KHI deux/ddl/Sig }\end{array}$ & & $\begin{array}{r}0,0 \\
0,9 \\
0,9 \\
0,9 \\
80,648 /\end{array}$ & .000 & \\
\hline
\end{tabular}

Tableau 1- Résultats de l'analyse factorielle confirmatoire de l'échelle de mesure de l'engagement envers la communauté

Les mesures de l'engagement envers la marque et de l'intention de fidélité à la marque ont également fait l'objet d'une validation par ACP suivie d'une analyse factorielle confirmatoire. L'ensemble des indices d'ajustement sont acceptables; il en est de même pour les indices de frabilité et de validité convergente. Les résultats de ces analyses sont présentés en annexe A2, le détail des items retenus après purification et validation sont présentés en annexe A3. 


\section{ANALYSE DES RESULTATS}

Cette première phase de validation des échelles de mesures autorise à passer à la phase de test des hypothèses.

Test de l'hypothèse Hl : Effet médiateur de l'engagement envers la communauté dans la relation entre l'intensité de participation à la CVM et l'intention de fidélité à la marque.

Nous testons ici l'hypothèse selon laquelle l'impact de l'intensité de participation du membre à la communauté virtuelle de marque sur son intention de fidélité à la marque se fera par l'intermédiaire de son engagement envers la communauté. En d'autres termes, l'intensité de participation à la communauté aurait un impact positif sur l'engagement envers cette communauté, ce dernier influençant alors à son tour l'intention de fidélité à la marque.

Afin de tester cet effet médiateur de l'engagement envers la communauté, deux modèles d'équations structurelles ont été construits. La démarche suivie est celle de Lam et alii (2004) qui adaptent le test de médiation de Baron et Kenny (1986) à l'analyse de données par équations structurelles. Cette démarche consiste alors à spécifier deux modèles : un premier modèle schématisant le lien direct entre la variable explicative (ici l'intensité de participation à la CVM) et la variable à expliquer (ici l'intention de fidélité à la marque) et un second modèle schématisant la relation de médiation (ici avec l'engagement envers la communauté).

Les résultats obtenus garantissent que l'ensemble des conditions qui permettent de vérifier l'existence d'une médiation sont réunies. Il est donc possible de conclure à l'existence d'une médiation totale de l'engagement envers la communauté dans la relation entre l'intensité de participation à la CVM et l'intention de fidélité à la marque puisque, comme le montre la figure 2 , le paramètre estimé pour le lien entre l'intensité de participation à la CVM et l'intention de fidélité à la marque, significatif dans le premier modèle, devient non significatif dans le second modèle lors de l'introduction de l'engagement envers la communauté comme médiateur. 


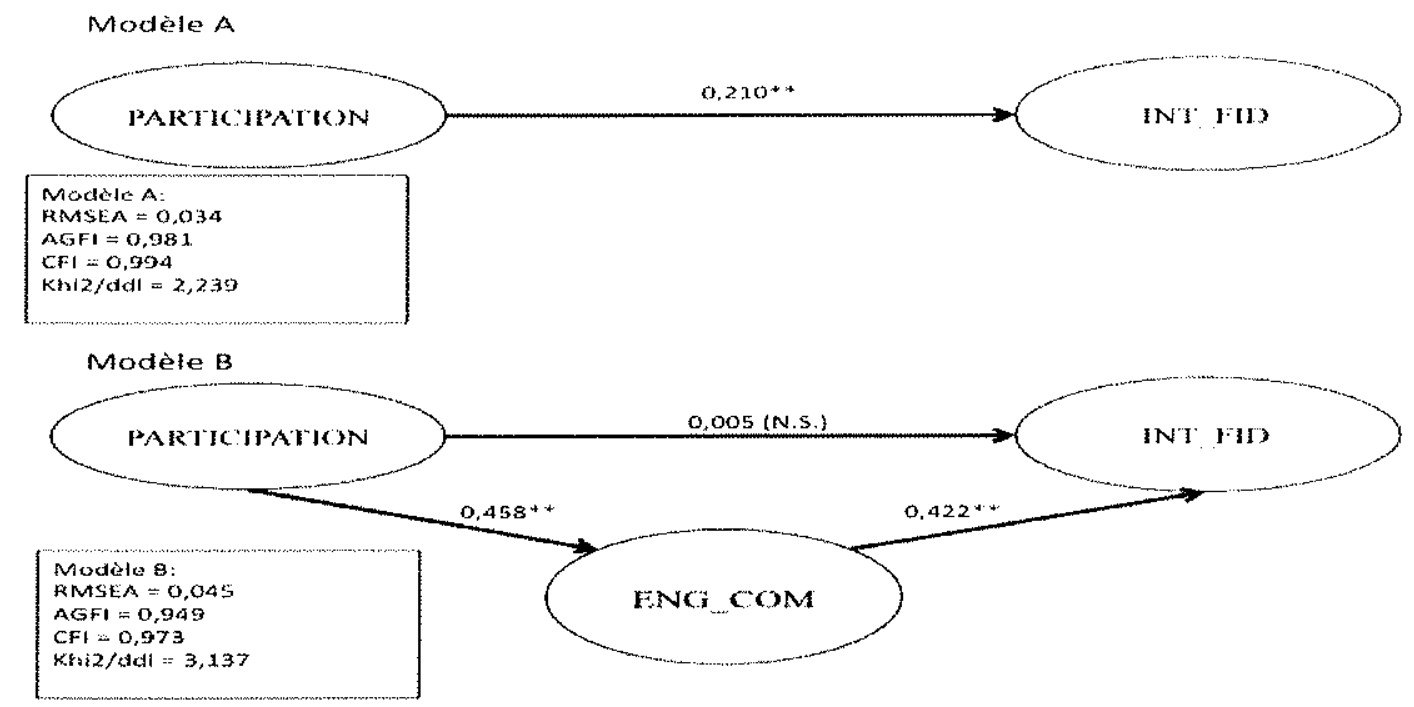

Figure 2 - Résultat du test de médiation de l'engagement envers la communauté sur la relation entre l'intensité de participation à la CVM et l'intention de fỉdélité à la marque

Test des hypothèses $\mathrm{H} 2$ et $\mathrm{H} 3$ à travers un modèle d'équations structurelles

Afin de tester l'hypothèse $\mathrm{H} 2$ postulant l'existence d'une relation bidirectionnelle réciproque entre l'engagement envers la communauté et l'engagement envers la marque, ainsi que l'hypothèse $\mathrm{H} 3$ qui postule l'impact de l'engagement envers la marque sur l'intention de fidélité à la marque, un modèle intégrateur prenant en compte toutes les relations du modèle théorique a été construit à travers un modèle global d'équations structurelles via le logiciel AMOS. Ce test permet de conclure aux bonnes qualités psychométriques du modèle sur la base des critères proposés par Hu et Bentler (1999) : RMSEA égal à 0,047, AGFI égal à 0,935 et CFI égal à 0,966 . Afin de pouvoir s'affranchir de l'hypothèse de multi-normalité des variables, un boostrap est effectué sur les données. La vérification de la significativité des paramètres estimés permet de conclure à la significativité de tous les liens structurels testés (voir figure 3 ). 


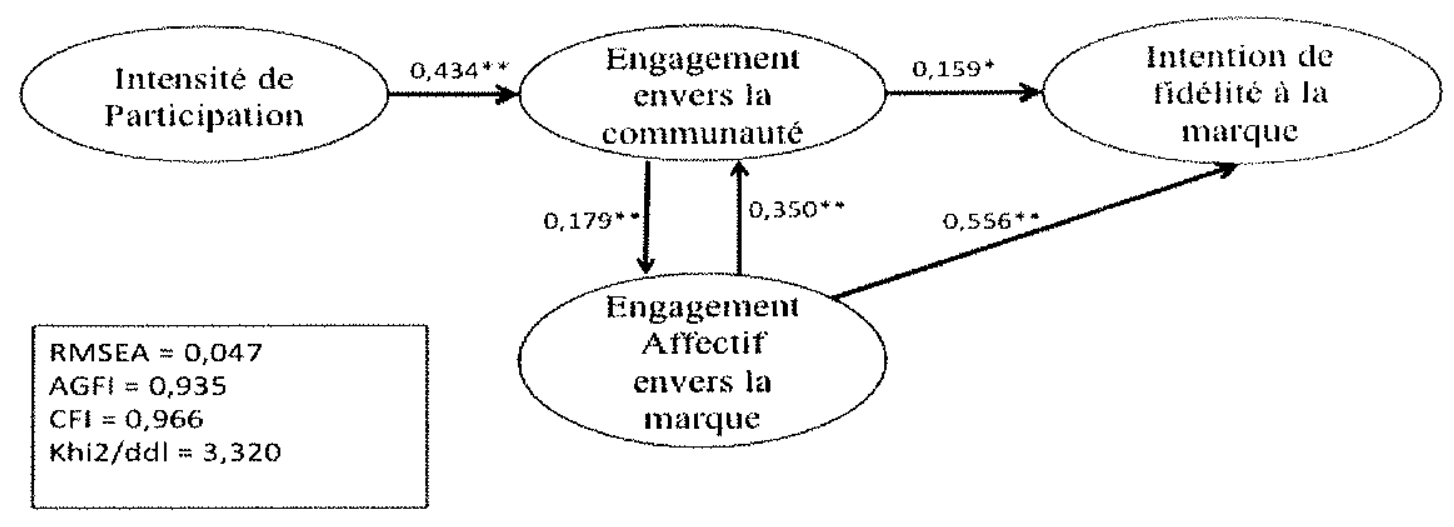

Figure 3 - Test du modèle global

Test de l'hypothèse H4 : Effet modérateur de l'ancienneté d'appartenance à la communauté :

Pour tester l'effet modérateur de l'ancienneté de l'appartenance, une analyse multi-groupes est effectuée selon les recommandations de Sauer et Dick (1993) grâce au module d'AMOS. Ainsi, après une première étape de test de différence de modèle (test de la significativité de la différence de Khi2 entre un modèle libre et un modèle contraint), qui a permis de conclure à l'existence d'une modération (voir Annexe A4), le sens de la modération est apprécié par l'analyse des valeurs des paramètres des relations structurelles du modèle pour chaque groupe (nouveaux $v s$ anciens) comme le précise le tableau 2.

Les résultats montrent un effet modérateur positif pour toutes les relations structurelles du modèle hormis la relation qui relie l'engagement envers la marque à l'engagement envers la communauté. En effet, si pour toutes les autres relations, le paramètre estimé est plus élevé pour les anciens membres que pour les nouveaux, allant dans le sens de l'hypothèse postulée du rôle modérateur positif de l'ancienneté, le sens de la modération est inversé pour le lien engagement envers la marque $>$ engagement envers la communauté qui est plus fort chez les nouveaux membres qu'il ne l'est pour les anciens. 


\begin{tabular}{|c|c|c|c|c|}
\hline \multirow[t]{2}{*}{ Relation étudiée } & \multicolumn{2}{|c|}{ Nouveaux membres } & \multicolumn{2}{|c|}{ Anciens membres } \\
\hline & Paramètre & Valeur $\mathrm{t}^{8}$ & Paramètre & Valeur $\mathrm{t}$ \\
\hline $\begin{array}{l}\text { Participation à la CVM -> Engagement envers } \\
\text { la communauté }\end{array}$ & 0,375 & 10,71 & 0,569 & 14,58 \\
\hline \begin{tabular}{|c|}
$\begin{array}{c}\text { Engagement envers la communauté }-> \\
\text { Intention de fidélité }\end{array}$ \\
\end{tabular} & 0,160 & 2,71 & 0,164 & 2,02 \\
\hline $\begin{array}{c}\text { Engagement envers la marque -> Intention de } \\
\text { fidélité }\end{array}$ & 0,551 & 10,80 & 0,558 & 7,64 \\
\hline $\begin{array}{c}\text { Engagement envers la communauté }-> \\
\text { Engagement envers la marque }\end{array}$ & 0,151 & 1,99 & 0,176 & 2,02 \\
\hline $\begin{array}{c}\text { Engagement envers la marque }->\text { Engagement } \\
\text { envers la communauté }\end{array}$ & 0,398 & 4,23 & 0,309 & 3,76 \\
\hline
\end{tabular}

Tableau 2 : Estimation des paramètres de la modération de l'ancienneté d'appartenance à la communauté

\section{Discussion des Résultats}

La première hypothèse testée stipule l'existence d'un effet médiateur de l'engagement envers la communauté sur le lien qui existe entre l'intensité de participation à la CVM et l'intention de fidélité à la marque. Cette hypothèse, qui défend l'idée que l'impact de la participation à la communauté de marque sur un comportement de fidélité envers la marque doit passer par un engagement envers la communauté, a bien été validée. Ce résultat va, d'une part, dans le sens de ceux mentionnés par Shang et alii (2006), pour qui participer activement à une communauté virtuelle autour d'une marque peut être considéré comme une activité impliquante pour le consommateur, activité qui aura pour conséquence un engagement fort envers la CVM. D'autre part, la relation positive trouvée entre l'engagement envers la communauté et l'intention de fidélité à la marque va dans le sens des résultats de Algesheimer et alii (2005), pour qui l'intention de continuer à être membre de la communauté, autrement dit l'engagement envers la communauté, a pour conséquence des intentions de comportement envers la marque.

De plus, les résultats du test de cette première hypothèse permettent de conclure à une médiation "totale» de l'engagement envers la communauté sur la relation étudiée. Plus précisément, cela confirme que l'effet de l'intensité de participation à la CVM sur l'intention de fídélité envers la marque passe forcément par une augmentation de l'engagement envers la communauté. L'engagement envers la communauté est donc une variable essentielle dans le lien qui s'établit entre participation à la CVM et intention de fidélité à la marque. Les implications managériales qu'un tel constat peut avoir seront mises en avant ci après.

\footnotetext{
${ }^{7}$ ll s'agit du paramètre estimé standardisé et boostrapé

${ }^{8}$ Obtenu en divisant le paramètre boostrapé par l'écart type. Pour que le paramètre soit significatif, la valeur $t$ doit être supérieure à $|1,96|$.
} 
Le test de la seconde hypothèse, qui défend l'existence d'une relation bidirectionnelle réciproque entre l'engagement envers la communauté et l'engagement envers la marque, a également révélé la significativité de la double relation. Ainsi, si un engagement plus fort envers la marque a pour conséquence un engagement plus fort envers la communauté $(\beta=0,350$; $p<0,05)$, l'engagement envers la communauté influence à son tour positivement l'engagement envers la marque $(\beta=0,179 ; \mathrm{p}<0,05)$. Les deux types d'engagement se nourrissent donc mutuellement et s'influencent réciproquement. Cette idée rejoint par ailleurs celle avancée par Kozinets dès 1999 , idée selon laquelle la relation que va développer le membre avec une communauté de consommation se construit autour de deux dimensions «non-indépendantes » et « interreliées »: la relation avec l'objet de rassemblement (ici la marque) et la relation avec la communauté $^{9}$. Enfin, au regard de la valeur des paramètres estimés, il semblerait que l'engagement envers la marque joue un rôle primordial dans la formation de cette double relation.

La troisième hypothèse postulant un impact positif de l'engagement envers la marque sur l'intention de fidélité à la marque a été, certes sans surprise, confirmée. Ainsi, un consommateur engagé envers la marque a plus tendance à avoir l'intention de racheter celle-ci et de faire un bouche-à-oreille positif envers cette dernière. Ce résultat, s'il n'apporte rien de nouveau à la littérature existante, confirme tout de même le rôle prédicteur important que joue l'engagement envers la marque pour la fidélité dans le contexte des communautés virtuelles de marque.

Enfin, le rôle modérateur positif de l'ancienneté, constaté pour la plupart des relations du modèle, est intéressant à souligner. En effet, il montre que la modération joue positivement pour les membres les plus anciens et qu'elle est en ce sens un facteur d'amplification de l'effet de la participation à la communauté sur l'intention de fidélité à la marque. Notons tout de même que le sens de cette modération s'inverse pour une relation en particulier : celle qui relie l'engagement envers la marque à l'engagement envers la communauté. En effet, cette relation est plus forte chez les nouveaux membres qu'elle ne l'est pour les anciens. Ainsi, nous constatons que, si l'impact de la participation à la CVM sur l'engagement envers la communauté ainsi que celui de l'engagement envers la communauté sur l'engagement envers la marque sont particulièrement plus forts pour les anciens membres (respectivement $\Delta \beta=+0,19$ et $+0,03$ ), l'impact de l'engagement envers la marque sur l'engagement envers la communauté est, quant à lui, plus fort pour les nouveaux membres $(\Delta \beta=-0,09)$. Ce résultat permet de conclure que, alors que pour les anciens membres, l'engagement envers la communauté est un élément primordial dans l'explication de l'impact de la participation à la CVM sur l'intention de fidélité à la marque, pour les nouveaux membres, c'est l'engagement envers la marque qui semble être l'élément déclencheur de la chaîne, probablement parce que leur engagement envers la communauté n'est pas encore très développé. Cette conclusion tend d'ailleurs à renforcer l'intérêt de l'examen de la double relation entre engagement envers la communauté et engagement envers la marque.

\footnotetext{
${ }^{9}$ "The formation of lasting identification as a member of a virtual community of consumption depends largely on two non-independent factors. First is the relationship that the person has with the consumption activity... The second factor is the intensity of the social relationships the person possesses with other members of the virtual community. The two factors will often be interrelated 》, (Kozinets, 1999, p.254).
} 
Ces résultats et éléments de discussion amènent, dans la conclusion qui suit, à souligner les contributions du présent travail et les implications managériales qui peuvent en découler.

\section{CONCLUSION}

Cette recherche s'inscrit dans une volonté de mise en avant de l'intérêt de l'étude des regroupements de consommateurs autour d'une marque en soulignant l'impact de ces regroupements sur les attitudes et comportements des consommateurs vis-à-vis des marques. Elle $a$, en ce sens, permis de démontrer l'effet positif de l'intensité de la participation d'un consommateur à une CVM sur son intention de fidélité à l'égard de la marque, premièrement au travers de l'augmentation de l'engagement envers la communauté qu'un niveau de participation et d'activité élevé engendre et, deuxièmement, au travers de la relation duale qui va s'installer entre cet engagement envers la communauté et l'engagement envers la marque.

\section{Les apports et contributions de la recherche}

Le modèle global présenté précédemment, et dont les indices d'ajustement ont permis de valider les hypothèses posées quant aux relations entre intensité de la participation, engagement envers la communauté, engagement affectif envers la marque et intention de fidélité, permet de mieux comprendre, au plan théorique, le processus relationnel qui existe entre la participation à une communauté virtuelle de marque et l'intention de fidélité envers cette marque.

En outre, le rôle médiateur de l'engagement, perçu comme une volonté de maintenir sa relation avec le partenaire et considéré d'un double point de vue, envers la communauté tout d'abord et envers la marque ensuite, n'avait, à notre connaissance, jamais été démontré dans le contexte des communautés virtuelles de marque.

De plus, cette recherche permet de valider empiriquement, par le biais de données quantitatives, l'existence et l'effet de la relation que construit un consommateur avec une communauté virtuelle de marque dans un contexte où la plupart des recherches se sont limitées à des propositions conceptuelles et/ou à de l'exploration qualitative.

Enfin, l'étude d'une relation réciproque, entre l'engagement envers la communauté et l'engagement envers la marque, à travers un modèle bidirectionnel non récursif, est rare dans les recherches en marketing ${ }^{10}$. Or, cette relation est particulièrement intéressante et indique clairement, dans le contexte des communautés virtuelles de marque, que ces deux variables s'auto-influencent. Il serait donc judicieux que des modèles de ce type soient plus souvent utilisés en sciences humaines et sociales (ainsi, la relation qui peut exister entre qualité perçue et satisfaction ou encore entre satisfaction et fidélité mériterait d'être éclairée de cette manière).

\footnotetext{
${ }^{10}$ Nous pouvons ainsi citer par exemple deux articles publiés dans des revues internationales classées utilisant les modèles non-récursifs avec une relation bidirectionnelle réciproque : Beerli et alii, 2004; Bagozzi et alii, 1980.
} 
D'un point de vue managérial, le constat d'une influence du niveau de participation à une communauté virtuelle organisée autour d'une marque sur la relation que le consommateur entretient avec la marque et notamment sur son intention de fidélité envers cette dernière, -ainsi que nous avons pu le démontrer précédemment--, implique, qu'une entreprise ou un modérateur qui souhaite impliquer les membres dans la vie d'une communauté doit veiller à stimuler leur intensité de participation à cette dernière en encourageant la fréquence et la durée des visites mais également en diversifiant la nature des activités dans lesquelles les membres pourront s'impliquer. Ceci peut se faire, par exemple, par le biais de l'organisation d'évènements spéciaux, de rencontres virtuelles ou « réelles », la proposition de différentes tâches autour de la consommation des produits de la marque, telles que des concours ou des votes.

D'autre part, toute entité soucieuse du développement d'une communauté virtuelle autour d'une marque doit veiller au maintien de la double relation qui existe entre l'engagement envers la communauté et l'engagement envers la marque. En effet, puisque ces deux types d'engagement se nourrissent mutuellement, il est important de faire en sorte que la boucle ne se brise pas en favorisant un type d'engagement aux dépens de l'autre. Cela pourra se faire en proposant aux membres des activités qui favorisent à la fois la cohésion du groupe et en même temps l'échange autour de la consommation de leur marque favorite. Nous pouvons, dans ce sens, donner l'exemple des "pixelistes » qui proposent des sorties photos, permettant ainsi à leurs membres de partager leur passion commune lors d'une journée d'échanges.

Pareillement, le constat d'un rôle modérateur de l'ancienneté met en avant l'importance de l'engagement envers la communauté pour les membres anciens. Dans cette optique, des systèmes permettant aux anciens de se sentir valorisés au sein de la communauté sont à encourager, comme par exemple des systèmes de points destinés à prendre en compte leur reconnaissance au sein du groupe. D'autre part, l'examen du mécanisme modérateur de cette variable démontre que l'engagement envers la communauté passe par un engagement envers la marque particulièrement important pour les nouveaux membres. Il est donc important de mettre en place des actions susceptibles d'éveiller l'intérêt de ces nouveaux membres en les intégrant le plus tôt possible aux activités du groupe afin d'augmenter leur engagement envers la CVM. Le modérateur joue alors un rôle primordial d'accompagnement des nouveaux, en leur expliquant les «codes » utilisés par la communauté et en valorisant chacune de leur participation aux activités du groupe.

Par ailleurs, cette recherche présentant l'intérêt d'avoir été menée dans un contexte réel de communauté organisée autour d'une marque par des consommateurs, elle a donc permis de mettre en avant le mécanisme d'influence de la communauté à travers le rôle primordial de l'engagement pour de «vrais» consommateurs d'une «vraie» marque (Nikon) au sein d'une " vraie» communauté créée par ces derniers («Les Pixélistes). En ce sens, elle contribue à apporter des éléments de réponse et d'éclairage sur les questions auxquelles font face aujourd'hui maintes entreprises quant à la position à adopter face à ces communautés ${ }^{11}$. En effet,

\footnotetext{
${ }^{11}$ Nous pouvons citer à titre d'exemple la mésaventure qu'a connue GAP en octobre 2010 lors du changement de son logo, décision qui n'a pas du tout plu à ses centaines de "fans" qui l'ont bien fait savoir. Après plusieurs semaines de manifestations négatives sur la toile, la communauté de consommateurs a cu raison de la décision
} 
il est évident qu'il devient de plus en plus complexe et difficile pour les entreprises d'arriver à communiquer efficacement avec ces consommateurs qui se détournent des discours purement commerciaux, voire font de la résistance par rapport aux modes de communication habituels (Bagozzi et Dholokia, 2006a). Elles se doivent alors de prendre en compte les communautés de consommateurs comme source d'information sur les attentes de ces derniers mais également comme moyen d'entrer en contact avec eux dans un contexte basé sur une égalité de pouvoirs entre consommateurs et entreprises (Cova et Carrère, 2002).

Un éventail d'options se présente alors aux entreprises, allant de la décision d'adopter une position passive en observant de loin ces regroupements jusqu'à la décision d'adopter une position pro-active en proposant un espace d'échange pour ses consommateurs. Mais quelle que soit la position choisie, elle devra s'assurer d'adopter une position transparente afin de garder la confiance de ses consommateurs et par conséquent leur engagement envers la communauté et envers la marque (Casalo et alii, 2007; Shang et alii, 2006). En effet, une entreprise qui sousestimerait l'importance d'une démarche éthique au sein de ces communautés (en postant par exemple de faux messages de membres fictifs), risquerait des retours fortement négatifs en cas de découverte de ce type d'activités par les consommateurs. Or, ces derniers se caractérisent aujourd'hui par une réelle sensibilité à l'éthique des entreprises (Canel Depitre, 2000) et ils ont, de plus, les moyens technologiques d'influencer très négativement l'image d'une marque en utilisant le buzz négatif sur Internet (Roux, 2007).

Cependant, malgré son intérêt théorique et managérial, cette recherche présente un certain nombre de limites dont certaines ouvrent des voies de recherche futures.

\section{Limites et voies ultérieures de recherche}

Tout d'abord, le modèle a été testé auprès d'une seule marque et d'une seule communauté. Il conviendrait donc de pouvoir confirmer ces premiers résultats auprès d'autres marques et d'autres types de communautés (par exemple initiées par la marque), de même qu'au travers de catégories de produits et services variés, afin d'améliorer leur validité externe. Toutefois, les premières analyses de données faites sur la base d'une autre communauté de passionnés de photographie «Nikon Passion» (qui a fait l'objet d'une autre étude de notre part, en cours de traitement) confirment les résultats obtenus dans la présente recherche.

D'autre part, les variables retenues ne correspondent qu'à une petite partie des problématiques qui pourraient être examinées dans ce contexte des communautés virtuelles de marque. A titre d'exemples, les effets médiateurs de la confiance envers la communauté et de la satisfaction envers celle-ci, ainsi que les effets modérateurs du type de communauté (initiée par l'entreprise $v s$. initiée par les consommateurs), du type de bénéfice recherché, de l'expertise des

stratégique de GAP qui s'est vu dans l'obligation de faire machine arrière en abandonnant son nouveau logo aux dépens de l'ancien. 
membres vis-à-vis de la catégorie de produit pourraient être explorés de manière tout à fait pertinente dans des recherches ultérieures.

Il est aussi possible de considérer qu'une démarche de développement d'une échelle de mesure de l'engagement envers la communauté, spécifiquement pensée dans l'optique de la communauté de marque, aurait été préférable et aurait peut-être abouti à des résultats différents. Il serait intéressant à l'avenir que des chercheurs s'emparent de cette question de construction d'échelles spécifiques au contexte doublement particulier d'Internet et des communautés.

Il aurait été par ailleurs préférable de pré-tester l'échelle d'engagement envers la communauté dans un contexte de communauté virtuelle de marque plutôt que dans celui d'une communauté d'intérêts. En effet, il se pourrait que la différence de type de communautés ait eu une influence sur les résultats du prétest de cette échelle. Nous pourrions le vérifier lors d'une prochaine étude, étude qui devrait, dans la mesure du possible, prendre en compte les comportements effectifs des internautes en termes de participation ainsi que leurs comportements effectifs en termes de fidélité envers la marque. 


\section{BIBLIOGRAPHIE}

Algesheimer R., Dholakia M. et Herrmann A. (2005), The social influence of brand community: Evidence from European car clubs, Journal of Marketing, 69, 3, 19-34.

Algesheimer R. et Dholakia M. (2006), Do customer communities pay off?, Harvard Business Review, 84, 11, 26-30.

Allen N. et Meyer J. (1990), The measurement and antecedents of affective continuance and normative commitment to the organization, Journal of Occupational Psychology, 63, 1-18.

Alon, A.T., et Brunel F. (2007), Dynamics of community engagement: the role of interpersonal communicative genres in online community evolutions, Research in consumer behavior, 11, 371-400.

Amine A. (1998), Consumer s' true brand loyalty: the central role of commitment, Journal of Strategic marketing, 6, 4, 305-19.

Amine A. et Sitz L. (2004), "How does a virtual brand community emerge? Some implications for marketing research." Cahiers de Recherche de l'IRG, Paris.

Aubert-Gamet V. et Cova B. (1999), Servicescapes: From modern non-places to postmodern common places, Journal of Business Research, 44, 1, 37-45.

Armstrong A. et Hagel J. (1996), The real value of on-line communities, Harvard Business Review, 74, 3, 134-41.

Arnould E. J. et Price L. L. (2000), Authenticating Acts and Authoritative Performances, in S. Ratneshwar, D.G. Mick et C. Huffman (coord.), The Why of Consumption, Contemporary perspectives on consumer motives, goals, and desires, London, Routledge, 140-63.

Bagozzi R. (1980), Performance and Satisfaction in an Industrial Sales Force: An Examination of Their Antecedents and Simultaneity, Journal of Marketing, 44, 2, 65-78.

Bagozzi R.P. et Dholakia U.M. (2006a), Open source software user communities: A study of participation in Linux user groups, Management Science, 52, 7, 1099-115.

Bagozzi R.P. et Dholakia U.M. (2006b), Antecedents and purchase consequences of customer participation in small group brand communities, International Journal of Research in Marketing, 23, 1, 45-61.

Baron R.M. et Kenny D.A. (1986), The moderator - mediator variable distinction in social psychological research: conceptual, strategic, and statistical considerations, Journal of Personality and Social Psychology, 51, 6, 1173-82. 
Beerli A., Martin J., et Quintana A. (2004), A model of customer loyalty in the retail banking market, European Journal of Marketing, 38, 1/2, 253-75.

Bhattacharya C.B. et Sen S. (2003), Consumer - company identification: a framework for understanding consumers' relationships with companies, Journal of Marketing, 67, 76-88.

Butler B., Sproull L., Kiesler S. et Kraut R. (2002), Community effort in online groups: Who does the work and why?, in S. Weisband et L. Atwater (coord.), Leadership at a distance, Erlbaum, $32 \mathrm{p}$.

Canel Depitre B. (2000), Développement Durable et Comportement Citoyen du Consommateur, $I^{e r}$ Congrès International des tendances du Marketing en Europe.

Casalo L., Flavian C. et Guinaliu M. (2007), The impact of participation in virtual brand communities on consumer trust and loyalty; The case of free software, Online Information Review, 31, 6, 775-92.

Casalo L., Flavian C. et Guinalliu M. (2008), Promoting Consumer's Participation in Virtual Brand Communities: A New Paradigm in Branding Strategy, Journal of Marketing Communications, 14, 1, 19-36.

Cova B. (1997), Community and consumption Towards a definition of the "linking value" of product or services, European Journal of Marketing, 31, 3, 297-316.

Cova B. et Carrère V. (2002), Gâre à vos marques, Du contre-pouvoir tribal sur le net, $2^{\text {ème }}$ Congrès sur les tendances du Marketing, Paris-Venise.

Cova B. et Pace S. (2006), Brand community of convenience products, new forms of customer empowerment : The case "my Nutella The Community", European Journal of Marketing, 40, 9, 1087-1105.

Cunnigham S.M. (1961), Customer loyalty to store and brand, Harvard Business Review, $39,127-137$.

De Almeida S.O., Mazzon J.A., et Dholakia U. (2008), The Effects of Belonging to Consumer-Managed and Firm-Managed Virtual Brand Communities: The Case of Microsoft XBOX, Latin American Advances in Consumer Research, 2, 203-04.

De Valck K. (2004), Virtual community of consumption: Networks of consumer knowledge and companionship, Thèse de Doctorat, Erasmus University, Rotterdam.

De Valck K., Langerack F., Verhoef P. et Verlegh P. (2007), Satisfaction with virtual communities of interest: Effect on members' visit frequency, British Journal of Management, 18, $3,241-56$.

Dholakia U. M. (1997), An investigation of some determinants of brand commitment, 
Advances in Consumer Research, 24, 381-87.

Duncan O., Haller O. et Portes A. (1968), Peer influences on aspirations: A reinterpretation, American Journal of Sociology, 74, 2, 189-237.

Ellonen H.K., Tarkiainen A. et Kuivalainen O. (2010), The effect of website usage and virtual community participation on brand relationships, International Journal of Internet Marketing and Advertising, 6, 1, 85-105.

Fornell C. et Larcker D. (1981), Structural equation models with unobservable variables and measurement error, Journal of Marketing Research, 18, 1, 39-50.

Fullerton G. (2005), The impact of brand commitment on loyalty to retail service brands, Revue canadienne des sciences de L'administration, 22, 2, 97-110.

Garbarino E. et Johnson M.S. (1999), The different roles of satisfaction, trust, and commitment in customer relationships, Journal of Marketing, 63, 2, 70-87.

Garnier M. (2006), Fidélité à un site Internet: influence des dimensions de l'engagement et rôle modérateur de variables individuelles liées à Internet. Application à un moteur de recherche, Thèse en Siences de Gestion, Université Pierre Mendès France, Grenoble.

Gupta S. et Kim H.W. (2004), Virtual Community: Concepts, Implications, and Future Research Directions, Proceedings of the Tenth Americas Conference on Information Systems, New York.

Gupta S. et Kim H. (2007), Developing the Commitment to Virtual Community: The Balanced Effects of Cognition and Affect, Information Resources Management Journal, 20, 1, 28-43.

Heehyoung J., Olfman L., Islang K., Joon K. et Kyungtae K. (2008), The Influence of OnLine Brand Community Characteristics on Community Commitment and Brand Loyalty, International Journal of Electronic Commerce, 12, 3, 57-80.

Hu, L., et Bentler, P. M. (1999), Cutoff criteria for fit indexes in covariance structure analysis: Conventional criteria versus new alternatives, Structural Equation Modeling, 6, 1-55.

Iwasaki Y. et Havitz M.E. (1998), A path analytic model of the relationships between involvement, psychological commitment, and loyalty, Journal of Leisure Research, 30, 2, 25680 .

Jarvis C., Mackenzie S. et Podsakoff P. (2003), A Critical Review of Construct Indicators and Measurement Model Misspecification in Marketing and Consumer Research, Journal of Consumer Research, 30, 199-218.

Johnson M.D., Herrmann A. et Huber F. (2006), The evolution of loyalty intentions, 
Journal of Marketing, 70, 2, 122-32.

Kang I., Lee K.C., Lee S., et Choi J. (2007), Investigation of online community voluntary behavior using cognitive map, Computers in Human Behavior, 23, 1, 111-26.

Kaplan A. M. et Haenlein M. (2010), Users of the world, unite! The challenges and opportunities of social medias, Business Horizons, 53, 59-68.

Kozinets R.V. (1999), E-tribalized marketing? The strategic implications of virtual communities of consumption, European Management Journal, 17, 3, 252-64.

Lam S.Y, Shankar V., Erramilli M.K., et Murthy B. (2004), Customer Value, Satisfaction, Loyalty, and Switching Costs: An Illustration From a Business-to-Business Service Context, Academy of Marketing Science Journal, 32, 3, 293-311.

Langerak F., Verhoef P., Verlegh P., et De Valck K. (2003), The effect of members' satisfaction with a virtual community on member participation, ERIM Report Series Research in Management.

Langerack F., Verhoef P., Verlegh P. et de Valck K. (2004), Satisfaction and Participation in Virtual Communities, Advances in Consumer Research, 31, 56-57.

Levine R., Locke C., Searls D., et Weinberger D. (1999), The Cluetrain Manifesto: The End of Business as Usual, Perseus Books Group.

Li, S.Y. et Hung K. (2006), Impacts of Virtual Community Participation on Consideration Set Size, Brand Switching Intention, and Brand Loyalty, American Marketing Association, 17, 161-163.

McAlexander J.H., Kim S.K. et Roberts S.D. (2003), Loyalty: the influences of satisfaction and brand community integration, Journal of Marketing Theory and Practice, 11, 4, 1-11.

Moorman C., Deshpande R., et Zaltman G. (1993), Factors affecting trust in market research relationships, Journal of Marketing, 57, 1, 81-101.

Morgan R.M. et Hunt S.D. (1994), The Commitment-Trust Theory of Relationship Marketing, Journal of Marketing, 58, 3, 20-38.

Muniz A.M. et O'Guinn T.C. (2001), Brand community, Journal of Consumer Research, $27,4,412-32$.

Odin Y. (1998), Fidélité et Inertie: clarification conceptuelle et test empirique, Université Pierre Mendès France, IAE Grenoble.

Oliver R.L. (1999), Whence consumer loyalty?, Journal of Marketing, 63, 4, 33-44. 
Pritchard M.P., Havitz M.E. et Howard D.R. (1999), Analyzing the commitment-loyalty link in service contexts, Journal of the Academy of Marketing Science, 27, 3, 333 48.

Rothaermel, F.T., et Sugiyama S. (2001), Virtual Internet communities and commercial success: individual and community-level theory grounded in the atypical case of TimeZone.com, Journal of Management, 27, 3, 297-312.

Roux D. (2007), Ordinary resistance as a parasitic form of action: a dialogical analysis of consumer/firm relations, Advances in Consumer Research, 34, 1-8.

Sauer Paul L. et Dick Alan (1993), Using Moderator Variables in Structural Equation Models, Advances in Consumer Research, 20, 1, 637-40.

Schau H.J. et Muniz A.M. (2002), Brand communities and personal identities: Negotiations in cyberspace, Advances in Consumer Research, 29, 344-49.

Schouten J.W. et Mcalexander J.H. (1995), Subcultures of Consumption - an Ethnography of the New Bikers, Journal of Consumer Research, 22, 1, 43-61.

Shang R.A., Chen Y.C. et Liao H.J. (2006), The value of participation in virtual communities on brand loyalty, Internet Research, 16, 4, 398-418.

Wang Y.C. et Fesenmaier D. R. (2004), Towards understanding members' general participation in and active contribution to an online travel community, Tourism Management, 25, $6,709-722$. 
Annexe Al - Résultats de l'analyse factorielle en composantes principales de l'échelle d'engagement envers la communauté

\begin{tabular}{|c|c|c|c|c|}
\hline Libellé des items & Communauté & $\begin{array}{c}\text { Poids } \\
\text { factoriels }\end{array}$ & Dimension & $\begin{array}{l}\text { Fiablilité : } \\
\text { alpha de } \\
\text { Crombach }\end{array}$ \\
\hline $\begin{array}{l}\text { Je me sens comme un «membre de la } \\
\text { famille» au sein de la communauté des } \\
\text { Pixelistes (COM AFF1). }\end{array}$ & 0.781 & 0.852 & \multirow{4}{*}{$\begin{array}{c}\text { Engagement } \\
\text { Affectif }\end{array}$} & \multirow{4}{*}{0,813} \\
\hline $\begin{array}{l}\text { Je ressens de l'attachement pour les } \\
\text { membres de la communauté des } \\
\text { Pixelistes (COM AFF2). }\end{array}$ & 0.751 & 0.830 & & \\
\hline $\begin{array}{l}\text { Je ressens un fort sentiment } \\
\text { d'appartenance pour la communauté des } \\
\text { Pixelistes (COM AFF3). }\end{array}$ & 0.697 & 0.792 & & \\
\hline $\begin{array}{l}\text { Pour moi, la communauté des } \\
\text { Pixellistes a une forte signification } \\
\text { personnelle (COM AFF4). }\end{array}$ & 0.673 & 0.763 & & \\
\hline $\begin{array}{l}\text { Il me serait difficile de trouver ailleurs } \\
\text { les informations que me procure le } \\
\text { forum des Pixelistes (COM_CAL1). }\end{array}$ & 0.884 & 0.924 & \multirow[b]{2}{*}{$\begin{array}{l}\text { Engagement } \\
\text { Calculé }\end{array}$} & \multirow[b]{2}{*}{0,875} \\
\hline $\begin{array}{l}\text { Le soutien apporté par les autres } \\
\text { membres de la communauté des } \\
\text { Pixelistes est très important à mes yeux } \\
\text { et j'aurais des difficultés à le retrouver } \\
\text { dans une autre communauté } \\
\text { (COM CAL2). }\end{array}$ & 0.751 & 0.702 & & \\
\hline $\begin{array}{l}\text { Toute personne qui possède un appareil } \\
\text { photo Nikon devrait participer aux } \\
\text { discussions du forum Pixelistes } \\
\text { (COM OBL1). }\end{array}$ & 0.842 & 0.878 & \multirow{2}{*}{$\begin{array}{l}\text { Engagement } \\
\text { D'obligation }\end{array}$} & \multirow[t]{2}{*}{0,916} \\
\hline $\begin{array}{l}\text { Afin d'assurer la survie de la } \\
\text { communauté, je ressens l'obligation de } \\
\text { continuer à participer aux discussions } \\
\text { des forums des Pixelistes } \\
\text { (COM_OBL2). }\end{array}$ & 0.648 & 0.655 & & \\
\hline Variance expliquée & \multicolumn{3}{|c|}{$75.338 \%$} & \\
\hline Indice KMO & \multicolumn{3}{|c|}{0.885} & \\
\hline Test de Sphéricité de Bartlett & \multicolumn{3}{|c|}{ Khi $2=3823.936 /$ ddI $=28 /$ Sig $=.000$} & \\
\hline
\end{tabular}


Annexe A2 - Résultat de l'analyse factorielle confirmatoire de l'échelle de l'intention de fidélité à la marque

\begin{tabular}{|c|c|c|c|c|}
\hline Item & $\lambda$ & $\begin{array}{c}\lambda \\
\text { bootstrap }\end{array}$ & $\begin{array}{c}\text { Ecart } \\
\text { type }\end{array}$ & Valeur $t$ \\
\hline $\begin{array}{l}\text { Intention de rachat de la marque } \\
\text { FID_RACH1 } \\
\text { FID_RACH } 2 \\
\text { FID_RACH3 }\end{array}$ & $\begin{array}{l}0,892 \\
0,945 \\
0,708\end{array}$ & $\begin{array}{l}0,891 \\
0,944 \\
0,712 \\
\end{array}$ & $\begin{array}{l}0,014 \\
0,012 \\
0,024 \\
\end{array}$ & $\begin{array}{l}63,642 \\
78,666 \\
29,666 \\
\end{array}$ \\
\hline Rhô de Jöreskog & \multicolumn{4}{|c|}{0,888} \\
\hline Validité convergente & \multicolumn{4}{|c|}{0,729} \\
\hline $\begin{array}{l}\text { Intention de recommander la marque } \\
\text { FID_RECOM } 1 \\
\text { FID_RECOM } 2\end{array}$ & $\begin{array}{l}0,752 \\
0,841\end{array}$ & $\begin{array}{l}0,752 \\
0,841\end{array}$ & $\begin{array}{l}0,038 \\
0,023\end{array}$ & $\begin{array}{l}19,789 \\
36,565\end{array}$ \\
\hline Rhô de Jöreskog & \multicolumn{4}{|c|}{$\mathbf{0 , 7 7 7}$} \\
\hline Validité convergente & \multicolumn{4}{|c|}{0,636} \\
\hline $\begin{array}{l}\text { RMSEA } \\
\text { AGFI } \\
\text { GFI } \\
\text { CFI } \\
\text { KHI deux/ddl/Sig }\end{array}$ & & $\begin{array}{r}0,0 \\
0,9 \\
0,9 \\
0,9 \\
13,373 / \\
\end{array}$ & .010 & \\
\hline
\end{tabular}




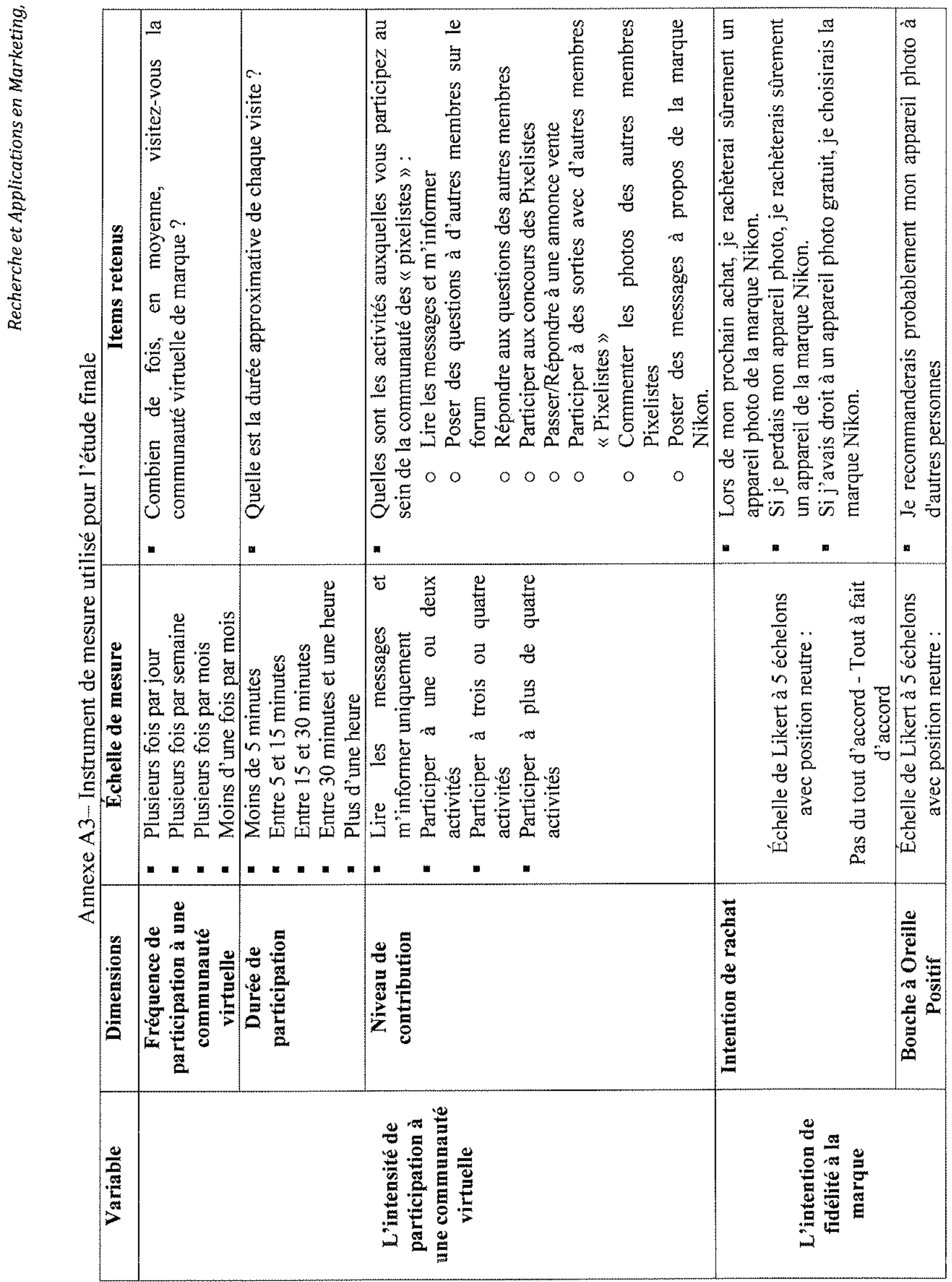




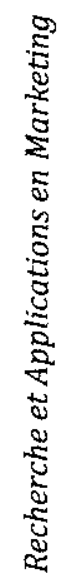

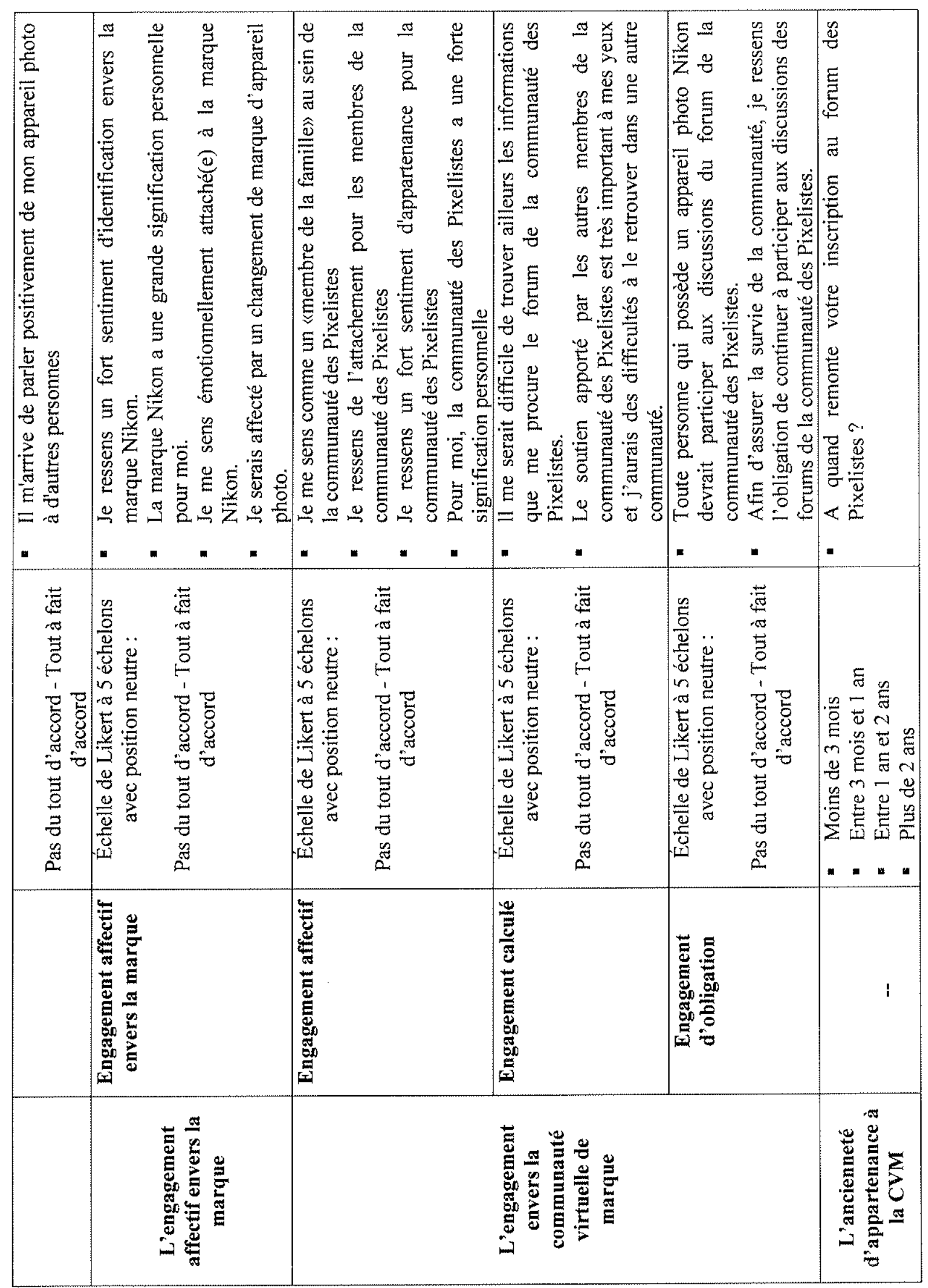

